\title{
基于苯乙烯腈结构的可逆力致变色化合物的合成及性能
}

\author{
毛文纲陈康欧阳密孙璟玮周永兵宋庆宝* 张诚* \\ (绿色化学合成技术国家重点实验室培育基地 浙江工业大学化学工程与材料学院 杭州 310014)
}

\begin{abstract}
摘要 合成了一种棒形的化合物 4,4'-二( $\alpha$-腈基-4-苯丙氧基苯乙烯)联苯(Ben-DCSB), 利用核磁共振(NMR)、质谱 (EI-MS)、傅里叶变换红外光谱(FT-IR)和元素分析等对其进行了结构表征. 对 Ben-DCSB 重结晶粉末进行研磨后, 其发 光颜色从蓝绿色变成黄绿色, 苂光量子效率 $\left(\Phi_{\mathrm{F}}\right)$ 从初始的 $52.7 \%$ 变为 $38.7 \%$, 表明该化合物具有力致变色性质. 扫描电 子显微镜(SEM)、X-射线衍射(XRD)和苂光寿命等测试结果显示, 这种现象是由于在外界环境刺激下改变了 Ben-DCSB 在聚集态下的分子堆积结构所造成的. 研磨后的样品暴露在溶剂蒸气(乙醇、二氯甲烷、四氢呋喃或丙酮)或 $100{ }^{\circ} \mathrm{C}$ 温 度下放置 $2 \mathrm{~min}$ 又能转换回初始状态的蓝绿色荧光, 表明化合物研磨后的样品具有气致和热致变色性能，且展现出可 逆变色性能. 对该化合物进行多次 “力-溶剂蒸气刺激” 和 “力-热刺激” 循环实验, 结果显示其具有很好的荧光可逆 转换性能. 热分析结果显示化合物 Ben-DCSB 在 $194{ }^{\circ} \mathrm{C}$ 和 $212{ }^{\circ} \mathrm{C}$ 间存在向列相(纹影织构)的液晶态; 其热分解温度为 $362{ }^{\circ} \mathrm{C}$, 表明该化合物具有较好的热稳定性.
\end{abstract}

关键词 力致变色; 可逆; 苂光材料; 分子堆积; 苯乙烯腈

\section{Synthesis and Characterization of New Cyanostilbene-Based Compound Exhibiting Reversible Mechanochromism}

\author{
Mao, Wengang \\ Chen, Kang Ouyang, Mi \\ Sun, Jingwei \\ Zhou, Yongbin \\ Song, Qingbao* Zhang, Cheng*
}

(State Key Laboratory Breeding Base for Green Chemistry Synthesis Technology, College of Chemical Engineering and Material Science, Zhejiang University of Technology, Hangzhou 310014, China)

\begin{abstract}
A rod-like molecules, 4,4'-bis( $\alpha$-cyano-4-phenproalkoxylstryl)biphenyl (Ben-DCSB), has been synthesized and characterized by nuclear magnetic resonance (NMR), electron ionization-mass spectrometry (EI-MS), Fourier transform infrared (FR-IR) and elemental analysis. Ben-DCSB presents excellent mechanochromism behaviors. Recrystallized blue-green Ben-DCSB powder are changed into relatively yellow-green with the fluorescence quantum efficiency $\left(\Phi_{\mathrm{F}}\right)$ obviously converting from original $52.7 \%$ to $38.7 \%$ after being ground. Photoluminescence (PL) spectra of the samples obtained under different conditions (ungrind and grind) show the maximum emission wavelength $\left(\lambda_{\mathrm{em}}\right)$ has a significant red-shift as high as $23 \mathrm{~nm}$ after grinding. Observing the morphological structure of Ben-DCSB under different aggregative state by using scanning electron microscopy (SEM), the results indicate that the damage on surface morphology which is caused by external stimuli can be restored by exposing ethanol vapor or heat treatment. The X-ray diffractometry (XRD) measurements, the ungrind dye displays indicative of well-defined microcrystalline-like structures, and grinding dye is amorphous feature in this state. These observations further indicate the mechanochromism originates from the altering mode of molecular packing from the high-order to disorder by grinding. Time-resolved fluorescence spectrofluorometer analysis of ungrind ande grind dye show the attenuation exponential increases from single exponential of ungrinding to double-exponential of grinding. The result further demonstrate order degree of the grind sample decreases. The ground powder return to original colour by fuming with solvent (ethanol, dichloromethane, tetrahydrofuran or acetone) or heating at about $100{ }^{\circ} \mathrm{C}$ for $2 \mathrm{~min}$. And the PL spectra show these samples can return original $\lambda_{\mathrm{em}}(485 \mathrm{~nm})$. Moreover, dye Ben-DCSB indicates good reversibility of fluorescence conversion upon grinding-fuming and grinding-heating processes. XRD measurements indicate that the amorphous sample obtained by grinding can revert to crystalline state by fuming with ethanol and heating. Furthermore, the thermal analysis results show Ben-DCSB exists liquid crystalline phase of nematic (schlieren texture) between $194{ }^{\circ} \mathrm{C}$ and $212{ }^{\circ} \mathrm{C}$, and thermal decomposition temperature of Ben-DCSB is $362{ }^{\circ} \mathrm{C}$, indicating that the compound has better thermal stability. Keywords mechanochromism; reversible; fluorescent material; molecular packing; cyanostilbene
\end{abstract}

\footnotetext{
*E-mail: qbsong@zjut.edu.cn and czhang@zjut.edu.cn; Tel.: 13186963759; 0086-0571-88320253

Received January 6, 2013; published February 20, 2013.

Supporting information for this article is available free of charge via the Internet at http://sioc-journal.cn.

Project supported by the National Key Basic Research Program of China (Nos. 2010CB635108, 2011CBA00700), International Sci \& Tech Cooperation Program, China (2012DFA51210) and the National Natural Science Foundation of China (Nos. 51203138, 51273179).

项目受国家重点基础研究发展计划(973 计划)前期专项项目(No. 2010CB635108, 2011CBA00700)、国际科技合作重点项目计划(No. 2012DFA51210) 及国家自然科学基金(Nos. 51203138, 51273179)资助.
} 


\section{1 引言}

近年, 有机固体荧光材料由于其在光功能材料中具 有很大应用潜力, 吸引了很多研究者的兴趣 ${ }^{[1 \sim 4]}$. 其中, 刺激响应型荧光转换材料(气致变色、热致变色和力致 变色材料)对于信息储存、光学记录和传感器的开发具 有重要意义 ${ }^{[5 \sim 7]}$.

力(压)致变色荧光材料是一种在不改变组成分子的 化学结构的情况下, 通过力刺激来改变分子堆积模式从 而实现固体荧光材料发光颜色转变的智能材料 ${ }^{[8,9]}$. 这 种调节分子堆积模式的方法可避免复杂的化学合成, 操 作简单. Weder ${ }^{[10]}$ 和 Park $^{[11]}$ 等课题组报道了基于苯乙烯 腈结构的材料在力刺激下由于材料堆积模式从一种有 序结构转变到另外一种有序结构而实现发光颜色的转 换. Harima 课题组 ${ }^{[12]}$ 最近证明力致变色现象的产生是由 于研磨过程中分子的堆积模式由初始的晶态转换为无 定形态. 此外, 近来, 一些有机荧光材料通过用力-加 热 ${ }^{[13 \sim 18]}$ 、力-溶剂/蒸气 ${ }^{[19 \sim 22]}$ 等外界刺激方式来可逆转换 材料荧光颜色或发光强度相继被报道. Chi ${ }^{[13,14]}$ 和 Wang 等 ${ }^{[15]}$ 课题组最近证明力致变色材料在研磨-加热过程中 其分子堆积模式能在晶态和无定形态之间可逆转换. Jia 课题组 ${ }^{[19]}$ 最近报道通过研磨一溶剂重结晶过程使材料在 无定形态和晶态之间可逆转换, 从而可逆调节发光材料 的荧光颜色和发光强度. Zhang 课题组 ${ }^{[21]}$ 报道证明了研 磨后的样品置于二氯甲烷的蒸气中 $2 \mathrm{~min}$, 能使无定形 态的样品可逆转换为晶态, 且多次研磨一二氯甲烷蒸气 过程, 可逆性能依旧良好. 然而, 由于在分子水平上对 力致变色的形成机理仍缺乏充分的理解, 到目前为止, 具有力致变色的有机小分子荧光材料还非常有限 ${ }^{[8,16]}$. 在已报道到的众多力致变色有机小分子苂光材料中, 具 有较高荧光量子效率的材料并不多见, 且具有较高荧光
量子效率的可逆力致变色性能的有机小分子荧光材料 还鲜有报道.

本课题组 ${ }^{[23]}$ 前期工作研究报道了基于苯乙烯腈-芳 胺结构的 CNS-4 不仅具有较强的分子内电荷转移能力 和聚集诱导发光性能, 而且也具有力致变色性能, 但我 们并未对其展开进一步的研究. 在本文中, 我们制备以 联苯二乙烯腈为核, 苯丙氧基苯基为封端的棒形结构的 有机发光材料 4,4'-二( $\alpha$-腈基-4-苯丙氧基苯乙烯)联苯 (Ben-DCSB)(如图 1 所示), 对 Ben-DCSB 的力致变色性 能及热致和气致外界环境因素影响下的荧光可逆转换 性质, 及其热性能进行了详细的研究.

\section{2 结果与讨论}

\section{1 力致变色性质}

Ben-DCSB 的重结晶固体粉末在 $365 \mathrm{~nm}$ 紫外光照 射下发出蓝绿色苂光 $\left(\Phi_{\mathrm{F}}=52.7 \%\right.$, 如图 $2 \mathrm{~A}$ 所示 $)$, 而对 样品进行研磨后, 粉末发出黄绿色的苂光 $\left(\Phi_{\mathrm{F}}=38.7 \%\right.$, 如图 2B 所示), 然后将上述研磨后的粉末置于乙醇、 $\mathrm{CH}_{2} \mathrm{Cl}_{2}$ 、四氢呋喃或丙酮蒸气中, 荧光颜色均能恢复到 初始的蓝绿光(如图 2C 所示), 同样将研磨后的样品 100 ${ }^{\circ} \mathrm{C}$ 热处理 $2 \mathrm{~min}$, 样品的苂光颜色也能回到初始的蓝绿 色(如图 2D 所示). 以上现象表明该化合物表现出力致 变色性能, 且也具备气致和热致可逆变色性能.

图 3 为化合物 Ben-DCSB 分别在 THF 溶液、研磨 前、研磨后、研磨后乙醇蒸气刺激及 $100{ }^{\circ} \mathrm{C}$ 热处理 $2 \mathrm{~min}$ 后样品的紫外可见光吸收光谱(A)和苂光光谱(B). 如图 3A 所示, 化合物 Ben-DCSB 在 THF 溶液状态下的最大 吸收峰为 $377 \mathrm{~nm}$; 研磨前的固体粉末在 $394.5 \mathrm{~nm}$ 处有 最大吸收峰, 较溶液的相比发生了 $17.5 \mathrm{~nm}$ 的红移, 是 由于在聚集态中, 分子堆积更加紧密, $\pi-\pi$ 作用加强, 从

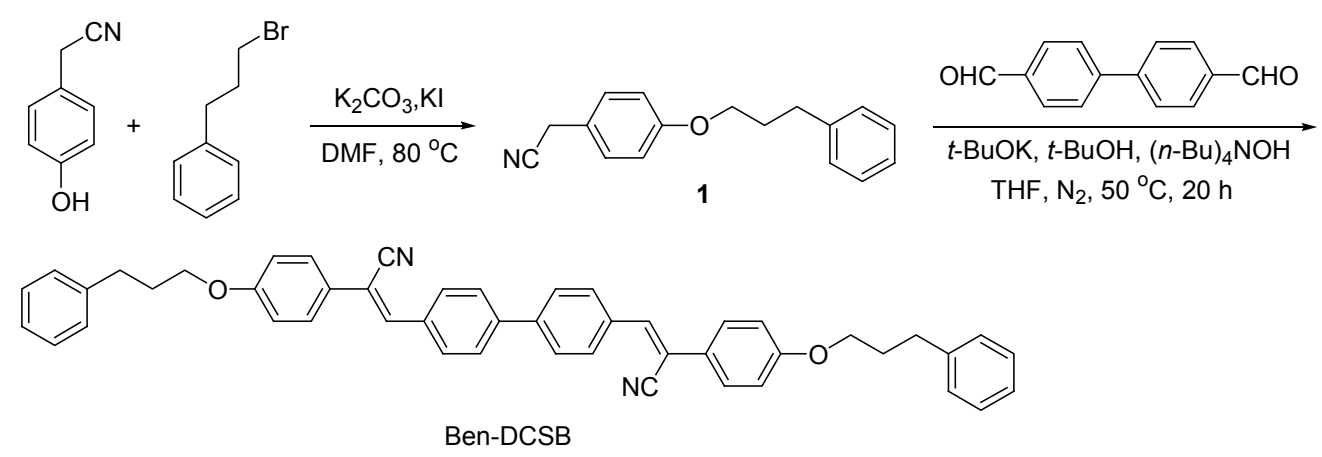

图 1 目标化合物 Ben-DCSB 的合成

Figure 1 Synthesis of the target compound Ben-DCSB

表 1 化合物研磨前与研磨后的物理性质

Table 1 Physical properties of ungrinding and grinding compound

\begin{tabular}{ccccccc}
\hline State & $\mathrm{Abs} \lambda_{\max } / \mathrm{nm} \mathrm{sol} / \mathrm{film}$ & $\mathrm{PL} \lambda_{\max } / \mathrm{nm} \mathrm{sol} / \mathrm{film}$ & $T_{\mathrm{m}}{ }^{a} /{ }^{\circ} \mathrm{C}$ & $T_{\mathrm{d}}{ }^{b} /{ }^{\circ} \mathrm{C}$ & $\tau / \mathrm{ns}$ & $\Phi_{\mathrm{F}}{ }^{c} / \%$ \\
\hline Unground & $377 / 394.5$ & $445 / 485$ & 212 & 362 & 1.96 & 52.4 \\
Ground & $-/ 389$ & $-/ 508$ & 211 & - & 1.98 & 38.7 \\
\hline
\end{tabular}

${ }^{a} T_{\mathrm{m}}$ : melting temperature; ${ }^{b} T_{\mathrm{d}}$ : decomposition temperature; ${ }^{c}$ fluorescence quantum yield of solid-state. 

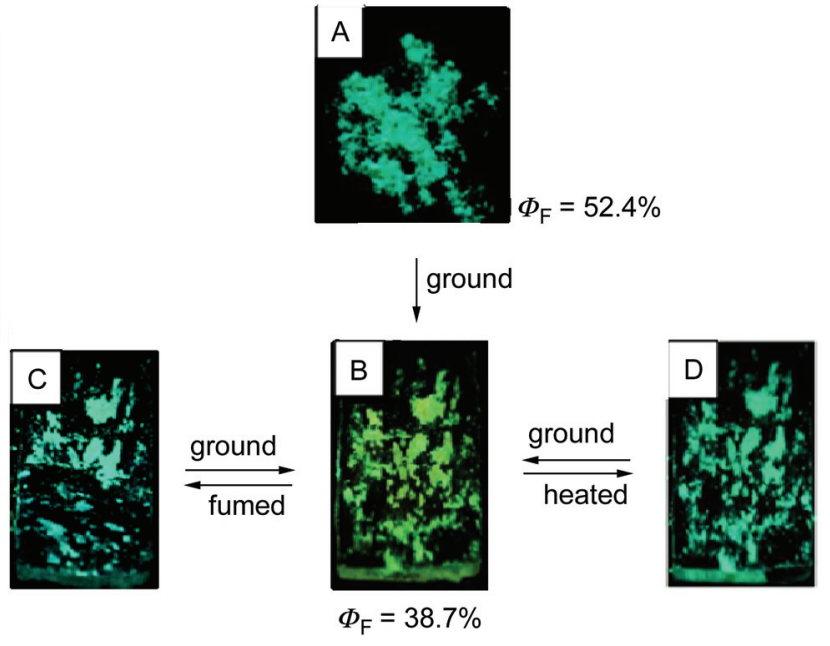

图 2 Ben-DCSB 的重结晶固体粉末在同状态下[研磨前(A)和研磨后 (B)]及研磨后的固体粉末(C)置于溶剂(乙醇、 $\mathrm{CH}_{2} \mathrm{Cl}_{2}$ 、四氢呋喃或丙 酮)蒸气刺激后或 $100{ }^{\circ} \mathrm{C}$ 热处理 $2 \mathrm{~min}(\mathrm{D})$ 的荧光图片(紫外灯 $365 \mathrm{~nm}$ 照射)

Figure 2 Photos of recrystallized Ben-DCSB powders in different states [unground (A), ground (B)] and ground powders are fumed with solvent (ethanol, $\mathrm{CH}_{2} \mathrm{Cl}_{2}$, tetrahydrofuran or acetone) (C) or heated at about 100 ${ }^{\circ} \mathrm{C}$ for $2 \mathrm{~min}(\mathrm{D})$ taken under $365 \mathrm{~nm}$ UV light

而发生红移; 在研磨后、乙醇蒸气刺激和 $100{ }^{\circ} \mathrm{C}$ 热处理 $2 \mathrm{~min}$ 后, 其最大吸收峰和研磨前基本上没有太大的变 化. 如图 3B 所示, 化合物 Ben-DCSB 在 THF 溶液状态 下的最大发射峰 $445 \mathrm{~nm}$, 同时在 $469 \mathrm{~nm}$ 处伴随一个明 显的肩峰; 研磨前的固体粉末相比于溶液发射光谱的肩 峰消失, 在 $485 \mathrm{~nm}$ 处有最大发射峰, 物质经研磨后, 发 射光谱发生了 $23 \mathrm{~nm}$ 的红移. 这种力刺激导致化合物的 苂光光谱发生红移可能是由于力刺激后分子间的距离 减小, 堆积的更加紧密, 偶极或者 $\pi-\pi$ 作用加强导致 的 ${ }^{24,25]}$. 而用乙醇蒸气刺激和 $100{ }^{\circ} \mathrm{C}$ 热处理 $2 \mathrm{~min}$ 后, 最大发射峰恢复到起始的峰位置.

如图 4A 所示, 利用 SEM 观察Ben-DCSB 不同聚集 态的形貌结构, 在 $\mathrm{CH}_{2} \mathrm{Cl}_{2}$ 溶液中重结晶获得不规则片 状晶体, 其表面相对光滑. 而研磨后的样品变成紧密的 无序层状且表面变得粗粘(图 4B). 经溶剂蒸气或热处理 后(图 4C, D), 粗粘的表面又恢复到初始状态. 以上结果 表明, 外力刺激对表面形貌造成的破坏, 可通过溶剂或 热退火处理得到修复. 这种外界环境刺激导致的物质表 面形貌变化可能是缘于分子堆积模式的变化 ${ }^{[11]}$. 为了 进一步证实上述猜想, 我们通过 XRD 测试化合物 Ben-DCSB 不同聚集态的堆积结构. 图 5 为化合物 Ben-DCSB 重结晶粉末和经过研磨、研磨后乙醇蒸气刺 激或 $100{ }^{\circ} \mathrm{C}$ 热处理 $2 \mathrm{~min}$ 后的 XRD 图, 结果表明研磨 前 Ben-DCSB 具有很好的结晶性, 分子的堆积是有序的; 经研磨后, 初始状态的结晶衍射峰消失, 只出现了一个 弥散峰, 表明样品研磨后为无定形态; 对研磨后的样品 分别置于乙醇蒸气氛围中和在温度为 $100{ }^{\circ} \mathrm{C}$ 下放置 2 min 后, 其衍射峰位置和初始态相似, 说明经溶剂蒸气
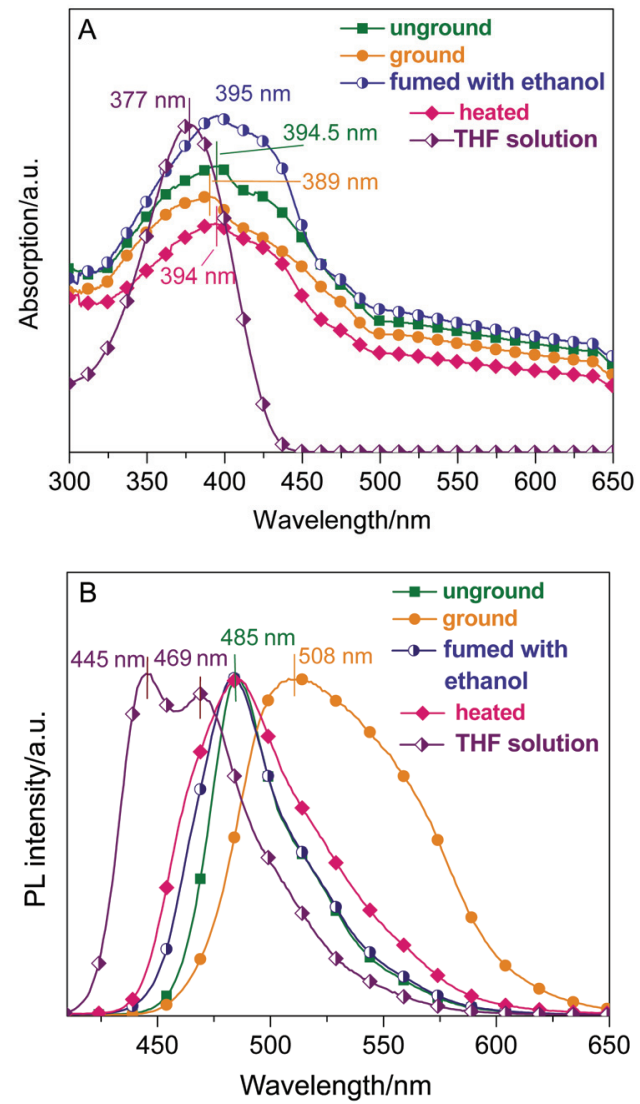

图 3 Ben-DCSB 分别在 THF 溶液、研磨前、研磨后、研磨后乙醇蒸 气刺激或 $100{ }^{\circ} \mathrm{C}$ 热处理 $2 \mathrm{~min}$ 后的紫外可见吸收光谱(A)和苂光光谱(B) Figure 3 The UV-vis (A) and PL spectra (B) of Ben-DCSB in different states (THF solution, unground, ground, grinding sample are fumed with ethanol or heated at about $100{ }^{\circ} \mathrm{C}$ for $2 \mathrm{~min}$ )

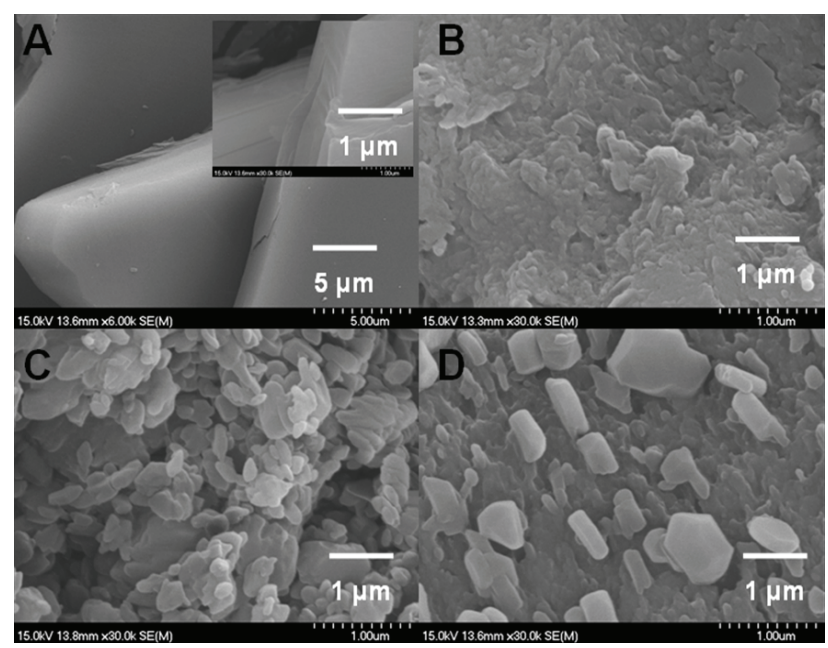

图 4 不同状下 Ben-DCSB 的 SEM 图: (A) 晶体; (B) 研磨处理后; (C) 样品 $\mathrm{B}$ 经过乙醇蒸气处理; (D) $100{ }^{\circ} \mathrm{C}$ 热处理 $2 \mathrm{~min}$ 后

Figure 4 SEM images of recrystallized Ben-DCSB powders in different states: (A) crystal; (B) ground; (C) sample B are fumed with ethanol; (D) heated at about $100{ }^{\circ} \mathrm{C}$ for $2 \mathrm{~min}$

和热刺激后, Ben-DCSB 分子从无定形态可逆转变为有 序的晶态. 以上结果表明, Ben-DCSB 样品经研磨后与 研磨前、溶剂蒸气及热刺激后相比呈现不同的 PL 光谱 
这是由于其聚集态下分子堆积结构不同造成的 ${ }^{[26 ~ 28]}$, 且该物质呈现出可逆力致变色性能. 如图 6 所示, 为研 磨前后 Ben-DCSB 的苂光衰减曲线, 结果显示研磨前激 发态以单指数的形式衰减, 衰减寿命 $\tau=1.96 \mathrm{~ns}$; 而经 过研磨后, 激发态以双指数的形式衰减, 衰减平均寿命 $\tau=1.98 \mathrm{~ns}$. 衰减指数增加也进一步说材料有序度的降 低, 这与 XRD 的结果相吻合 ${ }^{[12]}$.

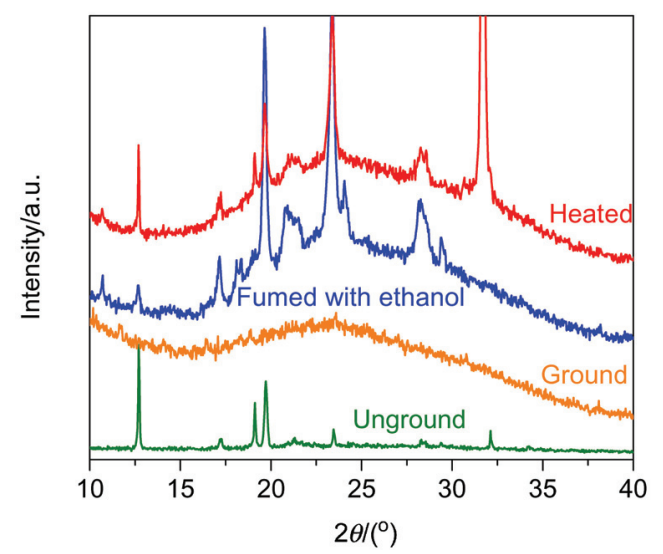

图 5 Ben-DCSB 在不同状态下(研磨前, 研磨后, 乙醇蒸气及加热处 理后)的粉末的 XRD 谱图

Figure 5 XRD patterns of Ben-DCSB in different states (unground, ground, fumed with ethanol and heated at about $100{ }^{\circ} \mathrm{C}$ for $2 \mathrm{~min}$ )

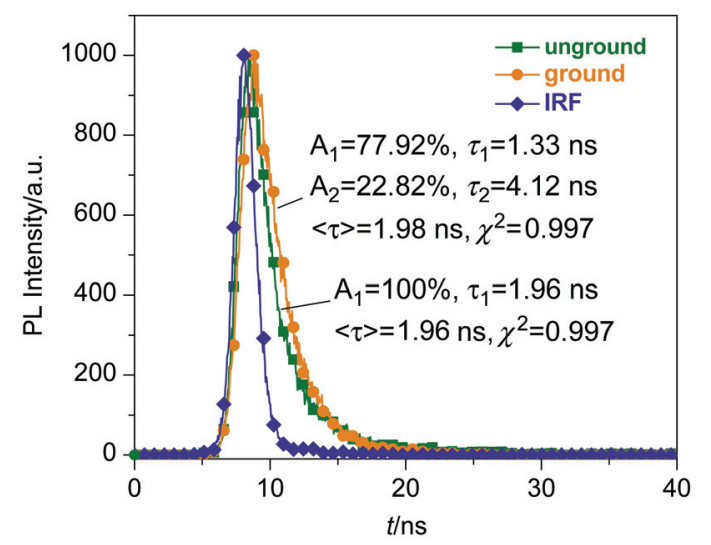

图 6 Ben-DCSB 在不同状态下(研磨前和研磨后)的荧光衰减曲线

Figure 6 Time-resolved emission decay curves of Ben-DCSB in different states (unground and ground)

\section{2 荧光可逆转换}

研磨后的Ben-DCSB 用溶剂蒸气或热处理使其恢复 到起始的发光颜色, XRD 证实其是从无定形态到晶态的 转变. 为了进一步测试材料的这种可逆的力致变色性能, 我们对其研磨后的样品进行多次 “力-溶剂蒸气” 和 “力一热” 循环试验. 将研磨后黄绿色荧光粉末(图 2B, $509 \mathrm{~nm}$ )置于乙醇的蒸汽氛围中或者滴加少量的乙醇溶 剂, 黄绿色荧光变成蓝绿色荧光, 发射峰的位置也恢复 到初始状态 $(485 \mathrm{~nm})$. 图 7A 为力-乙醇蒸汽多次循环刺 激后，其荧光发射峰的位置变化依然很明显，且在 485 $\mathrm{nm}$ 和 $508 \mathrm{~nm}$ 可逆转换. 除了乙醇蒸汽刺激影响外, 在 热(100 $\left.{ }^{\circ} \mathrm{C}, 2 \mathrm{~min}\right)$ 刺激下，物质的菼光也能恢复到初始 状态(485 nm). 如图 7B 所示, 通过力-热循环刺激后, 其荧光发射峰的变化也依然很明显且稳定，且在 $485 \mathrm{~nm}$ 和 $507 \mathrm{~nm}$ 可逆转换. 以上结果表明, 化合物 Ben-DCSB 同时具有良好的气致和热致可逆力致变色性能. 这种性 能将使得该化合物在多功能光学记录、信息存储和压力 传感器等应用领域有潜在的应用价值.
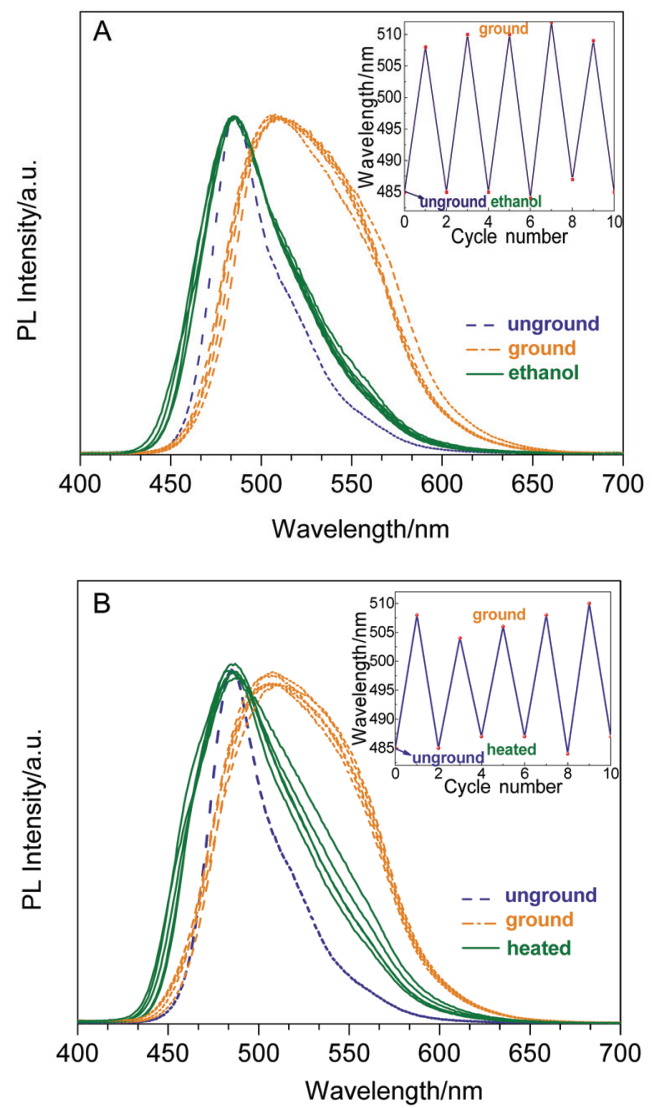

图 7 Ben-DCSB 在力-溶剂蒸气(A)和力-热(B)循环刺激下的 PL 光谱 ( $\mathrm{A} 、 \mathrm{~B}$ 中的插图为循环次数和最大荧光发射波长的关系图, 横坐标为 0 时表示未受力刺激; 为奇数时表示力刺激后, 偶数时表示恢复后(蒸 气熏或加热))

Figure 7 The PL spectra of Ben-DCSB in cyclic stimulus of grindingfuming (A) and grinding-heating (B) (insets: the reversible PL $\lambda_{\max }$ response over five consecutive cycles, when the abscissa is 0 , odd and even number, it represents Ben-DCSB in different states: 0 is unground, odd is ground and even is after recover (fuming or heated))

\section{3 热力学性质}

利用差热扫描量热分析(DSC)和热重分析(TG)对化 合物 Ben-DCSB 的热力学性质进行了研究, 所有测试均 在氮气环境中进行, 升温速率分别为 $5^{\circ} \mathrm{C} \cdot \mathrm{min}^{-1}(\mathrm{DSC})$ 和 $10{ }^{\circ} \mathrm{C} \cdot \mathrm{min}^{-1}$ (TG). DSC 分析结果(图 8)表明研磨后的 样品在 $174{ }^{\circ} \mathrm{C}$ 时出现一个冷结晶峰, 说明研磨后的化 合物是处于亚稳态，这与 XRD 分析结果相吻合. 化合 物在研磨前和研磨后熔化温度没有明显变化, 且均存在 两个吸热峰, 温度分别为 $194{ }^{\circ} \mathrm{C}$ 和 $212{ }^{\circ} \mathrm{C}$, 两个吸热峰 
间可能存在一个液晶相. 利用偏光显微镜测试观察到样 品在 $185{ }^{\circ} \mathrm{C}$ 为晶态, 而在 $198{ }^{\circ} \mathrm{C}$ 出现液晶态, 并在 205 ${ }^{\circ} \mathrm{C}$ 观测到典型的向列相纹影织构, 温度在 $220{ }^{\circ} \mathrm{C}$ 达到 清亮点(如图 9). 以上表明该物质 DSC 上存在两个吸热 峰是由于液晶相的存在. 如图 10 所示, 化合物 Ben-DCSB 经二氯甲烷重结晶的固体粉末在 $350{ }^{\circ} \mathrm{C}$ 之前 几乎没有重量损失, $5 \%$ 的重量损失时对应的温度 $\left(T_{\mathrm{d}}\right)$ 为 $362{ }^{\circ} \mathrm{C}$. 热重分析结果表明, 该物质有良好的热稳定性, 这将为制作热稳定性较好的器件创造条件.

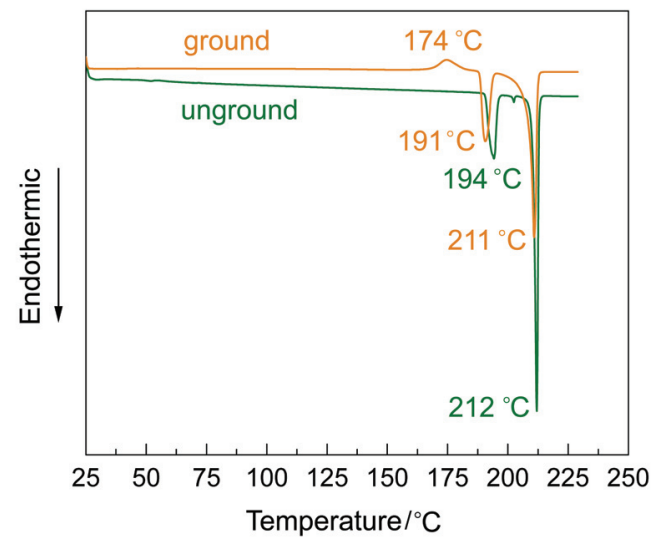

图 8 Ben-DCSB 在不同状态(研磨前和研磨后)的 DSC 曲线

Figure 8 DSC thermogram of Ben-DCSB in different states (before grinding and after grinding)

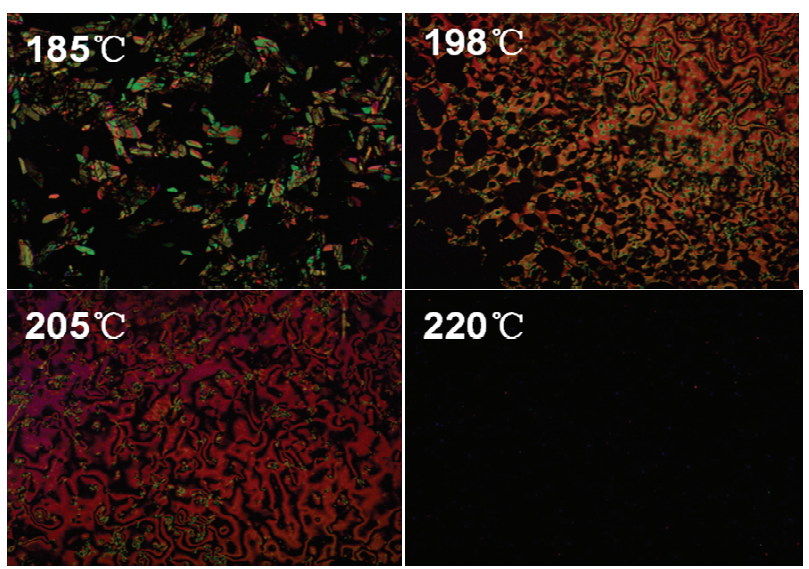

图 9 Ben-DCSB 在不同温度下观察的偏光显微镜图

Figure 9 Cross-polaried optical micrographs of Ben-DCSB in different temperatures

\section{3 结论}

合成了一个以联苯二乙烯腈为核, 苯丙氧基苯基为 封端的棒形结构有机发光材料 Ben-DCSB. 该化合物具 有明显的力致变色性质, SEM、XRD 和荧光寿命光谱等 测试结果表明, 力刺激改变了其聚集态下分子堆积结构, 从最初有序的晶态转变为无定形态, 导致其发光颜色及 发光强度的变化. 研磨后的样品进行溶剂蒸汽或热处理 后, 其发光颜色及发光强度恢复为研磨前, 且分子堆积 模式转变为晶态, 表现出可逆力致变色性能. 固体苂光

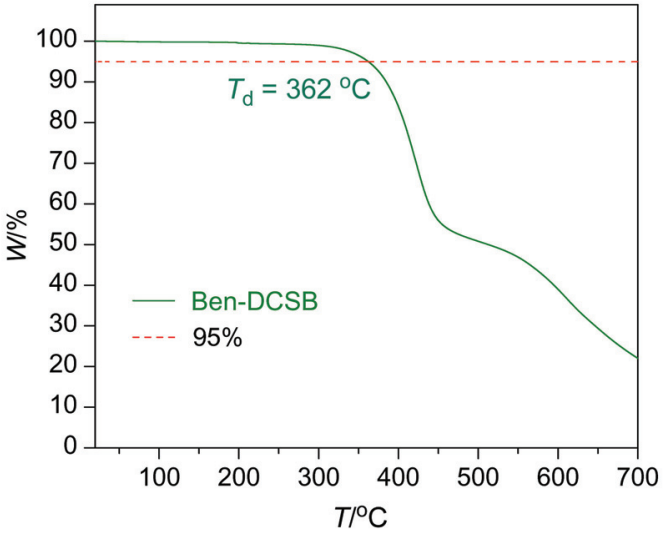

图 10 化合物 Ben-DCSB 的 TG 曲线

Figure 10 TG thermogram of Ben-DCSB

量子效率测试和多次 “力-热”与 “力-乙醇蒸气” 循环 刺激实验测试结果显示，该化合物具有较高的苂光量子 效率 $\left(\Phi_{\mathrm{F}}=52.7 \%\right)$ 和良好的苂光可逆转换性能. 热重分 析和差热扫描量热分析的测试结果表明该物质具有液 晶性和较好的热稳定性. 使该物质有望成为一种新型的 热稳定性较好的多功能智能材料.

\section{4 实验部分}

\subsection{4-(3-苯基丙氧基)-苯乙腈(1)的合成}

在 $50 \mathrm{~mL}$ 单口瓶中, 加入对羟基苯乙腈 $2.0 \mathrm{~g}$ (15 $\mathrm{mmol})$ 和 DMF $(20 \mathrm{~mL})$, 搅拌, 依次加入 $\mathrm{K}_{2} \mathrm{CO}_{3} 2.5 \mathrm{~g}(18$ $\mathrm{mmol})$ 和 $\mathrm{KI} 0.25 \mathrm{~g}(2.0 \mathrm{mmol})$. 油浴 $80{ }^{\circ} \mathrm{C}$ 下摚拌 $0.5 \mathrm{~h}$ 后, 用恒压滴定漏斗向反应容器中缓慢滴加 3-苯基溴丙 烷 $3.6 \mathrm{~g}(18 \mathrm{mmol})$ 的 DMF $5 \mathrm{~mL}$ 溶液, 反应放热, 滴加 完后, 溶液变为淡黄色. $80{ }^{\circ} \mathrm{C}$ 搅拌 $10 \mathrm{~h}$ 后, 冷却, 加入 $10 \mathrm{~mL}$ 蒸馏水淬灭反应，用二氯甲烷萃取，分出有机相， 用饱和食盐水洗涤 3 次, 无水硫酸镁干燥, 过滤, 浓缩 有机相, 得到的粗产物经柱层析分离, 正己烷为洗脱剂, 得到白色固体产物(分析数据详见 supporting information).

\section{$4.24,4^{\prime}-$ 二 ( $\alpha$ - 腈基 -4- 苯丙氧基苯乙烯 $)$ 联苯 (Ben-DCSB)的合成}

在 $100 \mathrm{~mL}$ 三口瓶中, 加入化合物 $11.5 \mathrm{~g}$ (6 mmol) 和 $15 \mathrm{~mL}$ THF. 搅拌, 向反应容器中依次加入 4,4'-联苯 二甲醛 $0.42 \mathrm{~g}(2 \mathrm{mmol}) 、$ 叔丁醇钾 $0.1 \mathrm{~g}(0.9 \mathrm{mmol})$ 、叔 丁醇 $15.5 \mathrm{~g}(138 \mathrm{mmol})$ 和 $(t-\mathrm{Bu})_{4} \mathrm{NOH} 1 \mathrm{~mL}(0.05 \mathrm{mmol})$. 在高纯氮保护下, $50{ }^{\circ} \mathrm{C}$ 油浴搅拌 $20 \mathrm{~h}$ 后, 停止加热, 冷 却, 向反应液中加入 $30 \mathrm{~mL}$ 蒸馏水, 有黄褐色的固体析 出. 抽滤, 得到的固体用 $80 \mathrm{~mL}$ 蒸馏水洗涤 3 次, 再用 $60 \mathrm{~mL}$ 无水乙醇洗涤 3 次, 干燥. 所得粗产物用二氯甲 烷重结晶, 得到黄绿色固体(分析数据详见 supporting information). 


\section{References}

[1] Kitai, A. Luminescent Materials and Applications, John Wiley \& Sons, Chichester, England, 2008.

[2] Fukazawa, A.; Yamaguchi, S. Chem-Asian J. 2009, 4, 1386.

[3] Entwistle, C. D.; Marder, T. B. Angew. Chem., Int. Ed. 2002, 41, 2927.

[4] Hudson, Z. M.; Wang, S. Acc. Chem. Res. 2009, 42, 1584.

[5] Toal, S. J.; Jones, K. A.; Magde, D.; Trogler, W. C. J. Am. Chem. Soc. 2005, 127, 11661 .

[6] Kishimura, A.; Yamashita, T.; Yamaguchi, K.; Aida, T. Nat. Mater. 2005, 4, 546 .

[7] Ning, Z.; Chen, Z.; Zhang, Q.; Yan, Y.; Qian, S.; Cao, Y.; Tian, H. Adv. Funct. Mater. 2007, 17, 3799.

[8] Sagara, Y.; Kato, T. Nat. Chem. 2009, 1, 605.

[9] Chi, Z. G.; Zhang, X. Q.; Xu, B. J.; Zhou, X.; Ma, C. P.; Zhang, Y.; Liu, S. W.; Xu, J. R. Chem. Soc. Rev. 2012, 41, 3878.

[10] Kunzelman, J.; Kinami, M.; Crenshaw, B. R.; Protasiewicz, J. D.; Weder, C. Adv. Mater. 2008, 20, 119.

[11] Yoon, S. J.; Chung, J. W.; Gierschner, J.; Kim, K. S.; Choi, M. G.; Kim, D.; Park, S. Y. J. Am. Chem. Soc. 2010, 132(39), 13675.

[12] Ooyama, Y.; Kagawa, Y.; Fukuoka, H.; Ito, G.; Harima, Y. Eur. J. Org. Chem. 2009, 31, 5321.

[13] Zhang, X. Q.; Chi, Z. G.; Zhang, J. Y.; Li, H. Y.; Xu, B. J.; Li, X. F.; Liu, S. W.; Zhang, Y.; Xu, J. R. J. Phys. Chem. B 2011, 115, 7606.

[14] Zhang, X. Q.; Chi, Z. G.; Xu, B. J.; Chen, C. J.; Zhou, X.; Zhang, Y.; Liu, S. W.; Xu, J. R. J. Mater. Chem. 2012, 22, 18505.
[15] Zhang, Z. L.; Yao, D. D.; Zhou, T. L.; Zhang, H. Y.; Wang, Y. Chem. Commun. 2011, 47, 7782.

[16] Luo, X. L.; Li, J. L.; Li, C. H.; Heng, L. P.; Dong, Y. Q.; Liu, Z. P.; Bo, Z. S.; Tang, B. Z. Adv. Mater. 2011, 23, 3261.

[17] Sase, M.; Yamaguchi, S.; Sagara, Y.; Yoshikawa, I.; Mutai, T.; Araki, K. J. Mater. Chem. 2011, 21, 8347.

[18] Zhang, G. Q.; Lu, J. W.; Sabat, M.; Fraser, C. L. J. Am. Chem. Soc. 2010, 132(7), 2160.

[19] Teng, M. J.; Jia, X. R.; Yang, S.; Chen, X. F.; Wei, Y. Adv. Mater. 2012, 9, 1255.

[20] Luo, J.; Li, L. Y.; Song, Y.; Pei, J. Chem. Eur. J. 2011, 17, 10515.

[21] Wang, C. G.; Chen, S. Y.; Wang, K.; Zhao, S. S.; Zhang, J. Y.; Wang, Y. J. Phys. Chem. C 2012, $116(33), 17796$.

[22] Dou, C.; Chen, D.; Iqbal, J.; Yuan, Y.; Zhang, H.; Wang, Y. Langmuir 2011, 27, 6323.

[23] Zhang, Y. J.; Sun, J. W.; Bian, G. F.; Chen, Y. Y.; Ouyang, M.; Hu, B.; Zhang, C. Photochem. Photobiol. Sci. 2012, 11, 1414.

[24] Ooyama, Y.; Ito, G.; Fukuoka, H.; Nagano, T.; Kagawa, Y.; Imae, I.; Komaguchi, K.; Harima, Y. Tetrahedron 2010, 66, 7268.

[25] Ooyama, Y.; Harima, Y. J. Mater. Chem. 2011, $21,8372$.

[26] Chen, J. W.; Law, C. C. W.; Lam, J. W. Y.; Dong, Y. P.; Lo, S. M. F.; Williams, I. D.; Zhu, D. B.; Tang, B. Z. Chem. Mater. 2003, 15, 1535 .

[27] Sagara, Y.; Mutai, T.; Yoshikawa, I.; Araki, K. J. Am. Chem. Soc. 2007, 129, 1520.

[28] Ouyang, M.; Yu, C. H.; Zhang, Y. J.; Hu, B.; Lü, X. J.; Sun, J. W.; Zhang, C. Acta Phys.-Chim. Sin. 2012, 28(12), 2944. (欧阳密，俞春 辉, 张玉建, 胡涁, 吕晓静, 孙璟玮, 张诚, 物理化学学报, 2012 , 28(12), 2944.)

(Cheng, B.; Fan, Y.) 